\title{
Taste of nutrients: Amino acids, vitamins, and fatty acids
}

\author{
SUSAN S. SCHIFFMAN \\ Department of Psychiatry, Duke University, Durham, North Carolina 27706 \\ and \\ CHARLES DACKIS \\ Duke University Medical Center, Durham, North Carolina 27706
}

\begin{abstract}
Multidimensional scaling techniques were used to determine the range in taste of nutrients for human subjects. The nutrients tested (amino acids, vitamins, and fatty acids) span the traditional sweet, sour, salty, and bitter gustatory quality range. The results also suggest that alkaline, sulfurous, and fatty qualities exist as well; the possibility that these three quality groupings are due to olfactory or tactile rather than gustatory input is discussed.
\end{abstract}

The traditional literature in gustation (Henning, 1916; von Skramlik, 1926) assumes that all gustatory stimuli are describable in terms of four "primary" qualities: sweet, sour, salty, and bitter. Henning proposed a geometric model in the form of a tetrahedron to describe the realm of gustatory quality. $\mathrm{He}$ arranged the four gustatory "primaries" at the corners of the tetrahedron, assuming that intermediate sensations fell on the edges and surfaces. Von Skramlik endorsed the sweet, sour, salty, and bitter primaries used by Henning because he found he could match the taste of inorganic salts by mixing appropriate amounts of each of the four primaries. Von Skramlik suggested that all other taste primaries, which have been considered historically in addition to sweet, sour, salty, and bitter are not gustatory but related to smell, touch, pain, and temperature.

The purpose of this study is to examine the taste qualities of nutrients (amino acids, vitamins, and fatty acids). The taste qualities of these stimuli are not well defined at the present time, since it is difficult to describe many of them phenomenologically by means of the sweet, sour, salty, and bitter primaries. This is most probably due to the fact that either (1) the taste of nutrients is not only due to gustatory input but is confounded by smell, touch, pain, and temperature components as well, or (2) the gustatory realm is not limited to the sweet, sour, salty, or bitter range. Since many nutrients cannot be ordered phenomenologically within the range of the four primaries, recently

This paper was supported in part by a grant from N.I.C.H.D. to the senior author, HD NS 06853; a grant from the National Science Foundation to R. P. Erickson, GB-33464X; and a grant from N.I.C.H.D. to the Center for Study of Aging and Human Development, HD 00668. developed multidimensional scaling procedures (Guttman, 1968; Lingoes, 1965; Schiffman \& Falkenberg, 1968) are employed here to arrange the nutrient stimuli in multidimensional spaces to more fully understand their range in quality.

Multidimensional scaling methods are quite helpful in understanding the quality range of sapid substances because they are open-ended so that all stimuli can be ordered; they do not require that the stimuli be describable by the sweet, sour, salty, and bitter categories. These scaling procedures can arrange the taste stimuli in a multidimensional space, so that those stimuli which are judged experimentally to be similar to one another are located close to each other in the multidimensional space; stimuli which are judged to be dissimilar are located far from each other in the space. At the very least, these procedures will reveal meaningful arrangements of the nutrient stimuli based on experimental measures. They may also be helpful in furthering our understanding of both the psychological and physicochemical dimensions in gustation.

Three major attempts have been made to order taste stimuli by means of the application of multidimensional scaling methods (Schiffman \& Erickson, 1971; Yoshida, 1963; Yoshida \& Saito, 1969) to human psychophysical data. Aside from Yoshida and Saito's results, which are somewhat ambiguous, the data indicate that the taste stimuli tested to date can be ordered in three dimensions. A rough tetrahedral structure, with sweet, sour, salty, and bitter at the corners, can be found in the three-dimensional arrangements. However, stimuli fall off the surfaces and edges of the tetrahedral structure- for example, potassium alum and monosodium glutamate (Yoshida, 1963) as well as 
alkaline substances, $\mathrm{NaOH}$ and $\mathrm{Na}_{2} \mathrm{CO}_{3}$ (Schiffman \& Erickson, 1971).

Whether these taste substances fall outside the tetrahedral prism solely on the basis of the gustatory rather than tactile or olfactory input has been investigated for the "alkaline taste" (Erickson \& Schiffman, in press; Schiffman \& Erickson, 1971). Erickson and Schiffman (in press) utilized single-unit gustatory neural data from the nucleus tractus solitarius in rat. They found that $\mathrm{NaOH}$ fell outside the tetrahedral structure on the basis of its neural code across many single gustatory units just as it did psychophysically in humans. Thus it appears that all gustatory experiences may not fall within the traditional categories of sweet, sour, salty, and bitter.

The specific purpose of this experiment is to understand the taste properties of 18 amino acids, 8 vitamins, and 4 fatty acids which are nutritionally important for the maintenance of human life. Multidimensional scaling techniques are applied to two sets of data: (1) ratings of these nutrients on semantic differential scales, which provide an ordering based on adjective descriptions of the stimuli, and (2) measures of similarity between nutrients taken two at a time, which avoid contamination of the ordering by verbal description. The spaces derived from these two methods are compared, and an attempt is made to understand the arrangements in the spaces with regard to both psychological and physicochemical parameters.

\section{METHODS}

\section{Subjects}

The subjects were 15 nonsmoking college students, without dentures and aged 19-22 years. who had not eaten within $2 \mathrm{~h}$ previous to testing.

\section{Stimulus Materials}

The stimulus materials were 30 nutrients important for the maintenance of human life. listed in Table 1, along with four additional standards: sweet (sucrose), bitter (quinine hydrochloride), salty $(\mathrm{NaCl})$, and alkaline $(\mathrm{NaOH})$. Vitamin $\mathrm{C}$ (ascorbic acid). which is a nutrient, was considered the sour standard. [The torms of the amino acids used are those employed by Buckley ${ }^{1}$ (unpublished) in an amino acid diet for allergic patients.) All stimuli (with the exception of $\mathrm{NaOH}$, which was diluted in distilled water to $.05 \mathrm{M} \mathrm{NaOH}$ because of its extreme causticity) were presented at room temperature $\left(72^{\circ} \mathrm{F}\right)$ in powdered or undiluted form and were reagent grade.

\section{Procedure}

The subjects were asked to place enough of each stimulus on the tongue to give an equi-intensive moderate taste for each nutrient. letting it come in contact with all parts of the mouth. In order to reduce olfactory input, each subject wore nose plugs and was asked not to inhale through the mouth while tasting a substance.

The experiment was divided into two parts: (1) judgments on a series of semantic differential scales which provide a verbal description of the stimuli, and (2) judgments of similarity which eliminate the need for verbal description.

For the semantic differential judgments, each of the 30 essential nutrients, as well as the standards, were rated on 46 semantic differential scáles: for example.

"good bad"

An " $x$ " was made along a 5-in. line from "good" to "bad." Forty-five scales, which had been used previously by Schiffman and Erickson (1971), were chosen for their range of description over the gustatory and somesthetic realm. "Tasteless-not tasteless" was added because some of the nutrients were found to be tasteless. The semantic differential judgments were made after the similarity judgments in order not to bias proximity measures with verbal descriptions.

For the judgments of similarity (which avoid contamination by verbal description), 21 stimuli (including sucrose, quinine hydrochloride, $\mathrm{NaCl}$, ascorbic acid, and $\mathrm{NaOH}$ as the sweet, bitter. salty. sour, and alkaline standards as well as 16 nutrients shown to range widely in quality on the basis of pretesting using semantic differential judgments) were compared, using all combinations of pairs, on the basis of the similarity of their tastes ( 210 comparisons per subject). Only 21 stimuli were used for similarity judgments because of the excessive number of comparisons required by inclusion of more stimuli. Most of the stimuli found to be relatively tasteless when pretested on the semantic differential scales were eliminated from this part of the experiment.

\section{Analysis of Data}

The similarity data were analyzed by the Guttman-Lingoes program. SSAI, a general nonmetric technique for finding the smallest Euclidean space for configuration of points (Guttman, 1968; Lingoes, 1965). This method takes as input similarity measures between stimuli, arranging the stimuli in a multidimensional space where the distances are related to the rank order of the experimental similarities.

The semantic differential ratings were analyzed by the Schiffman-Falkenberg method (1968). This method requires a rectangular matrix of the stimulus by adjective category form as input. The Schiffman-Falkenberg model assumes that the rectangular matrix, $R$. whose elements represent the relationship of a particular adjective to a particular stimulus, can be represented by two other matrices such that $\mathrm{R}=\mathrm{AS}$, where the A matrix is a set of row vectors describing adjectives and the $S$ matrix is a set of column vectors describing stimuli. The methodology reveals loadings for the stimulus and adjective vectors on dimensions of the same multidimensional space. The space is a vector space where the scaliar cross-product of a vector from the origin to an adjective with a vector from the origin to a stimulus is related to the experimental rating of that stimulus on that adjective. Interpretation of the dimensions is similar to interpreting factors in factor analytic solutions.

\section{RESULTS}

\section{Gustatory Space Based on Semantic Differential Data}

The results from the application of the Schiffman-Falkenberg multidimensional scaling procedure to semantic differential data are presented in Figure 1. This two-dimensional space, which accounts for $99 \%$ of the data, is a vector space; the scalar cross-product of a stimulus vector (from the origin to stimulus) with an adjective vector (from the origin to an adjective) is related to the relative importance of a given adjective in describing a given nutrient. Hence, adjectives and stimuli located far from the origin tend to be more powerful.

An examination of the relationship of the adjectives 
Table 1

A Description of the Stimuli Based on Semantic Differential Judgments for 15 Subjects

Stimulus Type Stimulus Description Based on Semantic Differential and Comments

Amino Acids Alanine

Arginine

Aspartic Acid

Cysteine $\mathrm{HCl}$

Glutamine

Glycine

Histidine $\mathrm{HCl}$

Isoleucine

Leucine

Lysine $\mathrm{HCl}$

Methionine

Phenylalanine

Proline

Serine

Threonine

Tryptophan

Tyrosine

Valine

Fatty Acids Linoleic Acid

Linolenic Acid

Oleic Acid
This is a good tasting substance which is sweet, flavorous, flat, and foodlike. The taste does not remain totally constant over time, however. Several subjects thought the aftertaste was similar to saccharin.

This is a sharp, alkaline, bitter, relatively constant taste which is described as strong, concentrated, and repulsive. It is a complex taste with minerally and salty elements, and is judged to be somewhat poisonous.

This is a strong, rapidly developing sour taste which is both tingling and sharp. Overall, it is less pleasant than ascorbic acid as well as being more dry.

Cysteine is a distinctly obnoxious tasting compound. It is strong. concentrated and nauseous; it was unitormly considered poisonous. Its rather complex, slowly developing taste was compared with sewage. rotten eggs and sulfur. No sour, sweet, or salty elements were noted by the subjects, although some found it to be bitter.

This is a soft, smooth substance with a sweetish, meaty taste. It is considered distinctly stale with a fatty element, and is somewhat unpleasant.

This is a sweet, pleasant, refreshing taste. It is described as simple and food-like; it is also considered very wet and smooth.

This is a complex, yet very clear taste with salty, sour, and bitter components. It is described as obnoxious. sharp. tingling. pungent, and extremely concentrated. It is definitely not foodlike.

This is a weak, tasteless compound with a flat, dry consistency. It is considered constant, not foodlike. and somewhat unpleasant. It is also rated as alkaline.

This is virtually indistinguishable from isoleucine.

Lysine is a somewhat complex substance which is salty with some bitter components. It is described as rapidly developing, sharp, and constant.

This is a very repulsive, metallic, minerally substance which some subjects judged to be bitter. It is dry. smooth, somewhat nauseous, and considered poisonous by over half of the subjects.

This is an obnoxious, minerally, metallic, bitter tasting substance. Its complex taste is sharp, thick, stale, concentrated, and dry. It has a pungent quality and also induces a strangling sensation.

This is a constant, rapidly developing, minerally, salty, sour substance. Although it is judged to be sharp, its complex taste is warming, flavorous, and not altogether unpleasant. Half the subjects detected a sweet quality in proline.

Serine has a constant, clear, sweet taste. It is also judged to be good, soft, smooth, and foodlike.

This is a moderately fatty, soft-textured substance. The taste is considered neither good nor bad by the subjects. Although it is not salty, sour, or bitter, it does have a slight sweetness.

This is a rapidly developing, sharp, bitter tasting substance. It is also labeled dry, minerally, stale, hard, and poisonous.

The most salient characteristic of this substance is its lack of any defined taste. It is not salty, sweet, sour, or bitter. It is described as soft, dry, flat, and stale.

This is a weak, dry, flat, minerally substance which is judged neither good not bad. It is judged to be unclear, leading to little agreement among the subjects. It is probably slightly bitter, with possible sour or sweet components. It is flat, constant, and unconcentrated.

This is a conplex, decidedly unpleasant, smooth, fatty tasting liquid which is rated strangling. nauseous. and slowly developing. It is also considered minerally, soapy, and pungent.

Linolenic acid has a taste very similar to linoleic acid except that it is not quite as unpleasant or soapy. It is also somewhat warming.

This is an unpleasant, smooth, fatty tasting liquid with a consistency slightly thicker than water. It is judged to be constant, and develops fairly rapidly. 
Table 1 continued

\begin{tabular}{|c|c|c|}
\hline Stimulus & Stimulus & Description Based on Semantic Diflerential and Comments \\
\hline & Palmitoleic Acid & $\begin{array}{l}\text { Itws substance is latty, mincrally, bitter, and repulsive. It is judged to be complex, having a hot, soapy } \\
\text { yuality. Lnlike oleic acid, this substance is judged pungent, burning, extremely strangling, and } \\
\text { puisonous. }\end{array}$ \\
\hline \multirow[t]{8}{*}{ Vitamins } & $\begin{array}{l}\text { Retinoic Acid } \\
\text { (Vitamin } A)\end{array}$ & $\begin{array}{l}\text { This is a weak, stale tasting chemical which has a fairly dry quality to it. Uniformly, it was not judged to } \\
\text { be sweet, sour, salty, or bitter. It is not particularly good or bad. Half the subjects found that it had a } \\
\text { definite taste, while the other half tended to judge it more tasteless. }\end{array}$ \\
\hline & $\begin{array}{l}\text { Thiamine } \mathrm{HCl} \\
\left.\text { (Vitamin } \mathrm{B}_{1}\right)\end{array}$ & $\begin{array}{l}\text { This is an extremely obnoxious sour-bitter substance which has strong, sharp, burning. bitey qualities as } \\
\text { well. It is judged to be clear, smooth, concentrated, and poisonous. It also has a pungent quality as well. }\end{array}$ \\
\hline & $\begin{array}{l}\text { Riboflavin } \\
\text { (Vitamin } B_{2} \text { ) }\end{array}$ & $\begin{array}{l}\text { This is a constant, extremely strong, bitter substance which is considered dry, minerally, and stale. } \\
\text { Although it has no burning qualities, it is consistently judged to be obnoxious. }\end{array}$ \\
\hline & Vitamin $B_{12}$ & $\begin{array}{l}\text { This is a practically tasteless red powder which has no salty, sweet, sour, or bitter qualities. It is thick, } \\
\text { stalc, dry, and that. }\end{array}$ \\
\hline & $\begin{array}{l}\text { Ascorbic Acid } \\
\text { (Vitamin C) }\end{array}$ & $\begin{array}{l}\text { This is a clear, strong, constant sour taste. Its flavorous taste develops rapidly and is described as } \\
\text { tingling, sharp, acidic and fruity. The subjects were divided on its hedonic quality, with half finding it } \\
\text { pleasant and half finding it somewhat unpleasant. }\end{array}$ \\
\hline & $\begin{array}{l}\text { Ergocalciferol } \\
\left(\text { Vitamin } \mathrm{D}_{2}\right)\end{array}$ & $\begin{array}{l}\text { This chemical is virtually tasteless. It is described as weak, flat, dry, thick, and stale, and is considered } \\
\text { neither pleasant nor unpleasant. }\end{array}$ \\
\hline & $\begin{array}{l}\text { Tocopherol } \\
\text { (Vitamin E) }\end{array}$ & $\begin{array}{l}\text { This is a very fatty, repulsive tasting, thick brown oil which, though obnoxious, is considered foodlike, } \\
\text { simple, constant, clear, and flat. One subject suggested that it bore a resemblance to concentrated } \\
\text { beef fat. }\end{array}$ \\
\hline & Vitamin $\mathrm{K}_{1}$ & $\begin{array}{l}\text { This is judged to be an almost tasteless, fatty substance. It is classified as soft, smooth, simple, and flat. } \\
\text { Two subjects found it moderately sweet. }\end{array}$ \\
\hline
\end{tabular}

and the nutrients tested reveals that the nutrients range widely in taste. Amino acids are found in all four quadrants of Figure 1; vitamins, in three quadrants; and fatty acids, in two quadrants. The projection of adjectives on the abscissa suggests that it is a hedonic dimension. The ordinate is not so readily interpretable. However, a dimension at a $45^{\circ}$ angle to the abscissa and ordinate from the first quadrant (upper right) to the third quadrant (lower left) tends to relate to the tactile character of the stimulus. The adjectives "sharp," "bitey," and "burning" fall toward one end of this $45^{\circ}$ dimension; "flat," "soft," and "smooth" fall toward the other end.

Since the arrangement of the adjectives and the nutrients involves some compromises. Table 1 is provided to give a more complete image of each stimulus based on raw semantic differential judgments. The descriptions in Table 1 were achieved by examining the individual data for all 15 subjects. If most subjects rated a substance as "sweet," it is described as "sweet" in Table 1. If there was wide variation over the subjects for a stimulus on an adjective, it was not considered a highly discriminating descriptor for that stimulus and thus is not included in Table 1.

\section{Gustatory Space Based on Similarity Judgments}

The three-dimensional arrangement of 21 stimuli achieved by application of Guttman's general nonmetric technique to similarity data can be seen in Figure 2. A three-dimensional solution was deemed appropriate after examining the coefficient of alienation or error, in maintaining the rank order of the experimental similarity judgments. The decrease in error from a one-dimensional (1D) solution through a five-dimensional (5D) solution for the nutrients and standards is: $1 \mathrm{D}-36 \% ; 2 \mathrm{D}-21 \% ; 3 \mathrm{D}-14 \% ; 4 \mathrm{D}-10 \%$; $5 \mathrm{D}-7 \%$. Since the four- and five-dimensional solutions do not appreciably decrease the error and since they do not reveal new relations among the stimuli from an examination of cross-sectional plots, a three-dimensional solution was considered appropriate for the similarity data.

Henning's prism accounts for part of the structure in Figure 2. Sucrose, glycine, serine, and alanine form the sweet "corner," while $\mathrm{NaCl}, \mathrm{QHCl}$, and ascorbic acid form the salty, bitter, and sour corners, respectively.

Falling off the side of the tetrahedron is a group (oleic acid, tocopherol, and glutamine) considered to have a fatty quality; cysteine $\mathrm{HCl}$, which contains sulfur; and arginine, which is described as alkaline. Whether "fatty" and "sulfurous" are definitely gustatory qualities rather than smell or tactile qualities must be determined in further experiments.

The placement of arginine in the space in Figure 2 


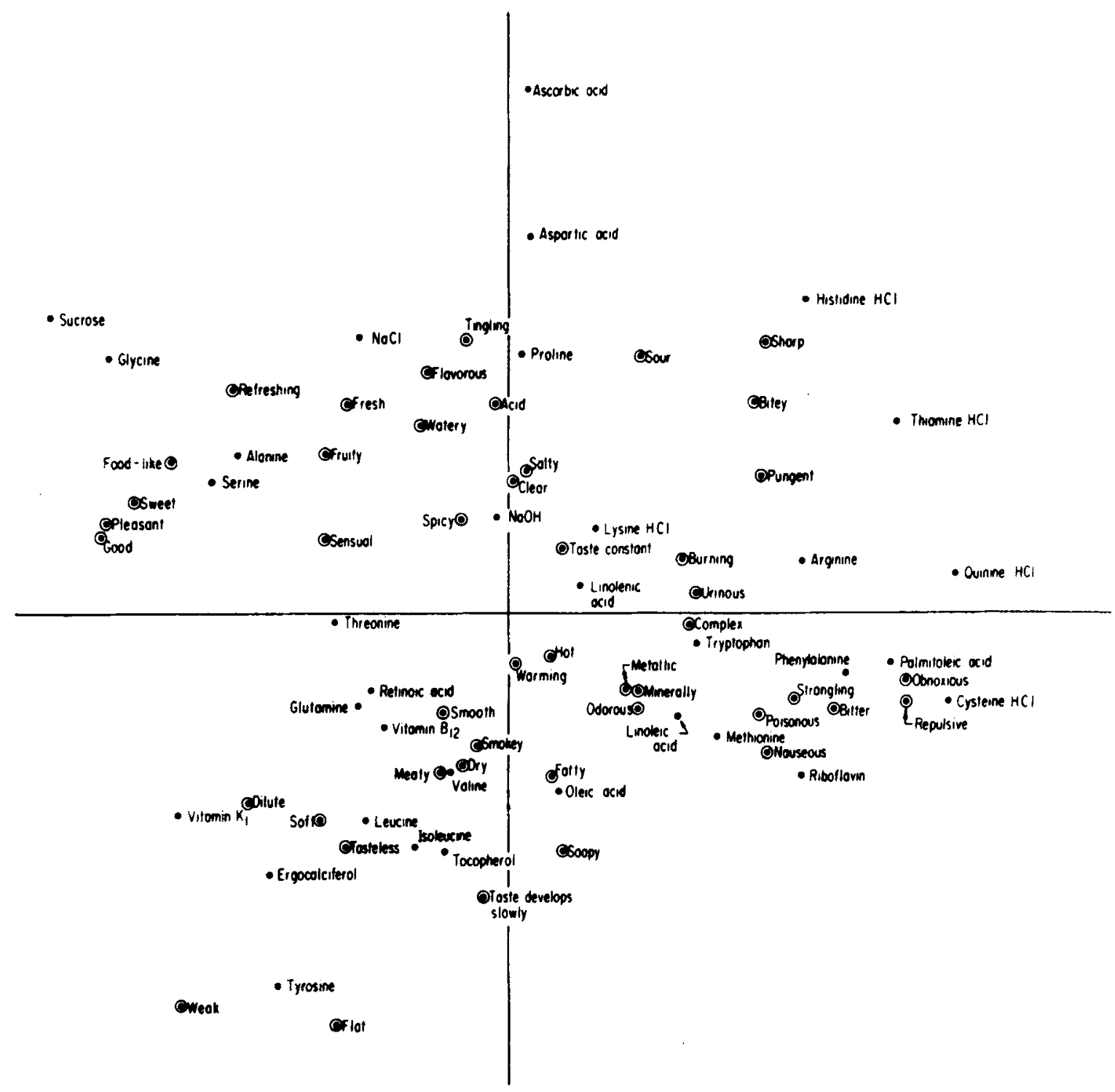

Figure 1. Space achieved by Schiffiman and Falkenberg's (1968) method for 30 nutrients and 4 standards using semantic differential judgments. This is a vector space. The scalar cross-product of vectors from the origin to a stimulus and to an adjective, respectively, is related to the relative importance of that adjective for that stimulus. Stimuli and adjectives falling far from the origin thus tend to be more powerful. The stimuli are labeled $\bullet$ and the adjectives are labeled $O$.

is similar to that for the alkaline stimuli, $\mathrm{NaOH}$ and $\mathrm{Na}_{2} \mathrm{CO}_{3}$, in the Schiffman and Erickson study (1971). $\mathrm{NaOH}$ in the present experiment, however, fell into the center of the space, toward the edge of the tetrahedron, rather than off to the side as it did in the Schiffman-Erickson experiment. This is probably due to the fact that $\mathrm{NaOH}$ was the only stimulus presented in this study in diluted liquid form and as such was rated equidistant from most of the stimuli. (Increasing the concentration of $\mathrm{NaOH}$ appreciably was deemed unwise by the experimenters due to its causticity.) Importantly, however, $\mathrm{NaOH}$ does not fall here with any of the "primary" groups. Also, it is located near arginine, which does fall out of the tetrahedral structure and is described as "alkaline."

\section{DISCUSSION}

\section{Appropriate Dimensionality for a Nutrient Solution}

The space with the appropriate dimensionality for the nutrients tested here must be considered to be the three-dimensional space in Figure 2, derived from the similarity measures, rather than the two-dimensional solution in Figure 1, derived from semantic differential data, since the dimensionality of the latter solution can be easily altered by the choice of 


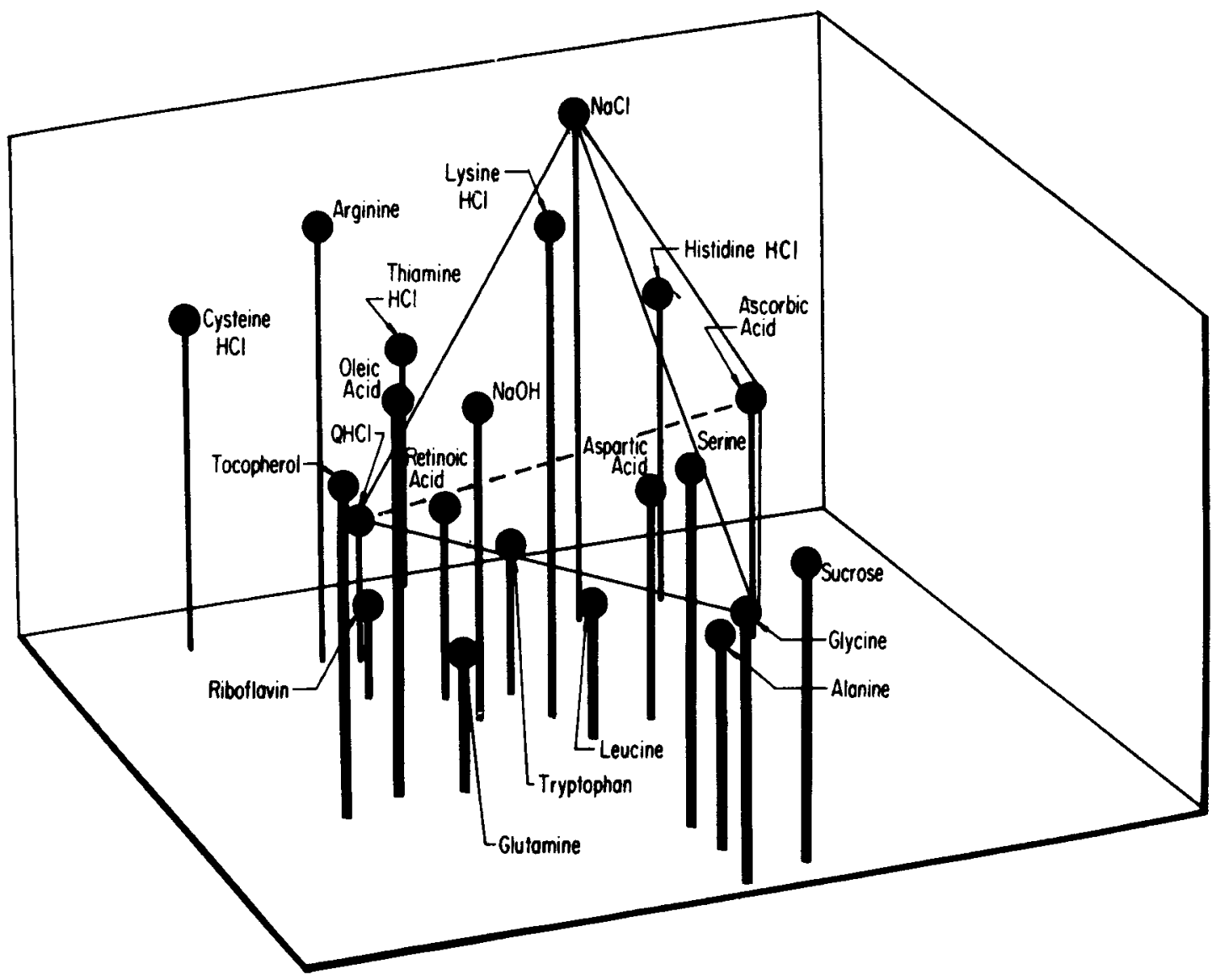

Figure 2. Space achieved by Guttman's (1968) method for 17 nutrients and 4 standards using similarity judgments. The interstimulus distances are related to psychological judgments of similarity. Stimuli judged to be similar are located near each other in the space.

adjectives. Schiffman and Erickson (1971) found that the inclusion or exclusion of adjectives used to describe a series of 19 compounds could alter the dimensionality of the space. This does not mean that the spaces based on semantic differential ratings are not useful, however, since they do illustrate meaningful relations among stimuli. Also, they are not as limiting in the number of stimuli which can be scaled as procedures which require similarity ratings for all pairs of stimuli.

\section{Psychological Range of Tacte Quality of Nutrients}

If the history of gustatory quality is correct, substances falling outside a tetrahedral structure should be related olfactory or tactile characteristics. In the introduction, however, new evidence (Erickson \& Schiff́man, in press; Schiffman \& Erickson, 1971), derived utilizing multidimensional scaling procedures, suggests that "alkaline" may be a gustatory quality in addition to the traditional sweet, sour, salty, and bitter qualities. This is due to the fact that $\mathrm{NaOH}$ falls outside a tetrahedral for both psychophysical data in humans and neural gustatory data in rats. Hence, the placement of the alkaline stimulus, arginine, outside the tetrahedron in Figure 2 may be due in part to gustatory input.

According to traditional theories, "fatty" and "sulfurous" are not gustatory but related to odor or tactile characteristics of the stimulus. In order to explore this view, multidimensional spaces for nutrients were found using two college students who were totally anosmic. (Both were thoroughly examined medically as well as tested in our laboratory with 20 trial substances.) The resultant space for the anosmics was almost identical to that in Figure 2 with the "nonprimary" qualities ("fatty," "sulfurous," and "alkaline") falling outside the tetrahedron.

Thus, it is suggested here that the placement of "fatty," "sulfurous," and "alkaline" substances outside the tetrahedral structure in Figure 2 is not related to olfactory input. Studies are presently being conducted with patients having Gasserian ganglion transsections to determine whether these "nonprimary" qualities are due to trigeminal input.

\section{Relationship of Physicochemical Properties to Taste} Based on both semantic differential judgments 
and similarity judgments, the following tentative conclusions can be made about the physicochemical properties of the essential nutrients employed in this study. The lighter weight amino acids (serine, alanine, and glycine) taste sweet, while the heavier ones tend to be bitter. [A molecular weight relationship in gustation was also found with other sapid substances by Schiffman and Erickson (1971).] Amino acids with acidic groups are sour. The three amino acids with basic groups (histidine HCL, lysine $\mathrm{HCl}$. and arginine) fall together in the first quadrant in Figure 1. even though histidine HCL and lysine $\mathrm{HCl}$ are crystalized from acid solutions-these three amino acids have in common salty, bitter, and sharp components. Amino acids with aliphatic side chains, including those containing $(\mathrm{OH})$ groups, tend to be either pleasant or tasteless. Those amino acids containing aromatic rings or sulfur tend to be unpleasant. None of the fatty acids was soluble in water; although water solubility has been considered important for stimulation in taste, every fatty acid was considered to have a fatty "taste" by all subjects. Other substances which are not soluble in water or which have low solubility (e.g., tyrosine, ergocalciferol, retinoic acid) tend to be tasteless. No specific trends were found for vitamins as a group.

\section{REFERENCES}

Erickson, R. P., \& Schiffman, S. S. The chemical senses: A systematic approach. In M. S. Gazzaniga and C. Blakemore (Eds.), Handbook of psychobiology. New York: Academic Press, in press.

Guttman. L. A general nonmetric technique for finding the smallest coordinate space for a configuration of points. Psychometrika, 1968, 33, 469-506.

Henning, $H$. The quality continuum of taste. Zeitschrift für Psychologie, 1916, 74, 203-219.

Lingoes, J. C. An IBM 7090 program for GuttmanLingoes smallest space analysis-I. Behavioral Science, 1965. 10. $183-184$.

Schiffman, H., \& Falkenberg, P. The organization of stimuli and sensory neurons. Physiology and Behavior, 1968, 3, 197-201.

Schiffman, S. S., \& ERIckson, R. P. A psychophysical model for gustatory quality. Physiology and Behavior, 1971, 7, 617-633.

Von Skramlik, E. Handbuch der Physiologie der niederen Sinne, I, Die Physiologie des Geschmackssinne. 1926. Pp. 346-520.

Yoshida, M. Similarity among different kinds of taste near the threshold concentration. Japanese Journal of Psychology, $1963,34,25.35$.

Yoshida, M., \& SaIto, S. Multidimensional scaling of the taste of amino acids. Japanese Psychological Research, 1969, 11, 149-166.

\section{NOTE}

1. C. E. Buckley, Duke Medical Center, Durham, North Carolina.
(Received for publication May 1, 1974; revision accepted September 11, 1974.) 